\title{
Costos ABC/ABM como herramienta de gestión empresarial. Caso empresa PlanERP Cía. Ltda.
}

\section{ABC/ABM Cost Management as a Business Excellence Tool. PlanERP Company Case.}

Maritza Elizabeth Torres Flores. ${ }^{1}$, Cecilia Ivonne Narváez Zurita, ${ }^{2} J u a n$ Carlos Erazo Álvarez, ${ }^{3} \&$ Jorge Edwin Ormaza Andrade. ${ }^{4}$

\section{DOI: https://doi.org/10.33262/visionariodigital.v3i2.1.549}

\begin{abstract}
.
Nowadays, companies face sudden changes in their production systems, resulting mainly from globalization and technological advances. Hence, on a worldwide scale is a tendency to incorporate management tools aimed to generate reliable information on the production costs and the support for strategic and tactical decision making. Consequently, the industry of software production requires a management tool. The idea is to provide accurate information for priority setting and focus on cost reduction and strategic planning to compete successfully in the business world.

This research proposes an ABC/ABM Cost Management Model for the Company PlanERP in the city of Cuenca, Ecuador. The aim is to foster the advancement of scientific knowledge related to production costs determination among companies that are deploying software and its use on decision making.

This study applied the experimental approach based on the case methodology. The first phase studied the most important variables in the management costs, and at the same time, the context of the software industry was considered as an essential part of this study. The second phase analyzed the processes of software implementation and features that simplify the description of the process map. The third phase described all the characteristics of the proposed model and its application. Finally, the last paragraph sets out the conclusions of this research.
\end{abstract}

Keywords: Activity Based Costing (ABC), Activity-Based Management (ABM), Decision Making, Costs, Competitiveness.

\footnotetext{
${ }^{1}$ Universidad Católica de Cuenca, Posgradista de la Maestría en Contabilidad y Auditoría, Universidad Católica de Cuenca, Ecuador, metorresf560@psg.ucacue.edu.ec

${ }^{2}$ Universidad Católica de Cuenca, Departamento de Posgrado, Cuenca, Ecuador, inarvaez@ucacue.edu.ec

${ }^{3}$ Universidad Católica de Cuenca, Departamento de Posgrado, Cuenca, Ecuador, jerazo@ucacue.edu.ec

${ }^{4}$ Universidad Católica de Cuenca, Departamento de Posgrado, Cuenca, Ecuador, jormaza@ ucacue.edu.ec
} 


\section{Resumen.}

En la actualidad todas las empresas independientemente de cual sean sus actividades buscan alcanzar mayor rentabilidad, crecimiento y permanencia en el mercado; tomando en cuenta que los beneficios no solo son para los accionistas sino para todos los interlocutores de las empresas, dentro de los cuales se destacan sus trabajadores, la comunidad, entre otros.

Una nueva cultura organizativa se va alcanzando paulatinamente en las empresas, para tratar de adaptarse al nuevo entorno que les rodea, con el fin de aumentar sus posibilidades de mercado, ocurren cambios en el exterior por lo que también, es necesario realizar cambios en el interior mediante la utilización de nuevas herramientas administrativas como es el caso del sistema ABC/ABM.

En este artículo se presenta la propuesta de un modelo de gestión de costos $\mathrm{ABC} / \mathrm{ABM}$, sustentado en identificar las actividades que realiza la organización, los recursos que consume, centro de costo y la cantidad de los recursos que es demandada por cada proceso hasta el producto o servicio final; y así, poder determinar el costo por actividad y costo final.

Para el desarrollo del modelo se inició con una fase de análisis de la literatura referente al tema, posteriormente se realizó un diagnostico en Empresa PlanERP Cía. Ltda., de la provincia Azuay, Ecuador, posteriormente se estableció un análisis de los resultados, un esquema del modelo y finalmente se describió cada uno de los componentes.

Palabras claves: ABC (Sistema de Costeo Basado en la Actividad), ABM (Gestión Basada en la Actividad), gestión, toma de decisiones, costos.

\section{Introducción.}

Las empresas de forma gradual implementan cambios para adaptarse al progreso de la sociedad y a las exigencias de mercados más competitivos y complejos. Uno de esos cambios es la utilización de alternativas más eficientes para la obtención de información financiera útil y confiable.

Es así que, a mediados de la década de los años 80, surgieron los costos ABC (Activity Based Cost System). Los primeros autores en tratar esta temática fueron Cooper Robin y Kaplan Robert, quienes propusieron un nuevo sistema de costos y una gestión basada en actividades (ABC/ABM); en vista de que el costeo tradicional no cumplía con las expectativas y necesidades de los empresarios. Tal deficiencia se debía principalmente a que el enfoque tradicional se basaba en la valoración de inventarios y en la determinación de costos de venta, lo cual proporcionaba información de los estados financieros limitada. Mientras que, el sistema de costos ABC (Activity Based Cost System o Costos Basados en Actividades), permite conocer con mayor precisión los causantes del costo, analizar todas las actividades 
de la empresa y establecer los costos de forma más acertada. Además, es considerado como una herramienta gerencial, para administrar las actividades y procesos de la empresa, así como para la toma de decisiones estratégicas, tácticas y operacionales.

Uno de los objetivos de los Costos $\mathrm{ABC} / \mathrm{ABM}$ es identificar todas las actividades que consumen recursos (inclusive las que no están directamente relacionadas), y asignarlos de manera adecuada y exacta a los diferentes procesos. Esto permite obtener información financiera confiable y relevante que es el sustento para la planificación y toma de decisiones (Mayorga, 2015). En consecuencia, el sistema de costos ABC no solo asigna o permite conocer los costos del proceso productivo, sino también, permite identificar cuál de los productos es más rentable para la empresa, entendiéndose como producto un bien o servicio (Pérez, 2009).

Por su parte, Rodriguez y Macarro (1996) indican que los costos ABC tienen como objetivo la excelencia empresarial, a través de la implementación de sistemas de calidad total; y, por consiguiente, la eliminación de actividades y reducción de ciclos que no generen valor, en este sentido, el sistema de costes ABC permite, además, conocer qué proceso o actividad desperdicia recursos, a efectos de tomar medidas correctivas oportunamente. $\mathrm{Al}$ respecto, Rodríguez (2008) considera que los costos ABC, mejoran la asignación de los recursos a los productos y permiten medir el desempeño de las actividades que se realizan en la empresa, efectuando una medición desde diferentes aspectos tales como: actividades, procesos, áreas de responsabilidad y productos.

Sin embargo, es necesario considerar que el sistema de costos $A B C$ se concentra en la asignación y optimización de los costos, descuidando la visión general de la empresa. La implementación de este sistema requiere o exige una capacitación y una gran concentración de esfuerzo para lograr una ejecución satisfactoria, y demanda gran parte de los recursos en las fases de diseño y producción (Baldera, 2016). En consecuencia, la implementación de este modelo requiere una inversión elevada.

\section{Aspectos teóricos de los costos ABC.}

El sistema de costos $\mathrm{ABC}$ determina los costos de las actividades basado en el uso de los recursos y establece costos a los objetos de costos, de acuerdo al consumo de cada una de las actividades; por ello, es importante considerar lo siguiente:

- Identificación de recursos.

- Identificación de las actividades.

- Asignación de inductores de costo asociados con cada actividad.

- Centro de Costos.

- Asignación de costos a los productos multiplicando la tarifa de inducción de costo por el volumen de unidades consumidas conductoras de costo por el producto. 
Figura 1. Pasos para la implementación de un sistema de Costos ABC.

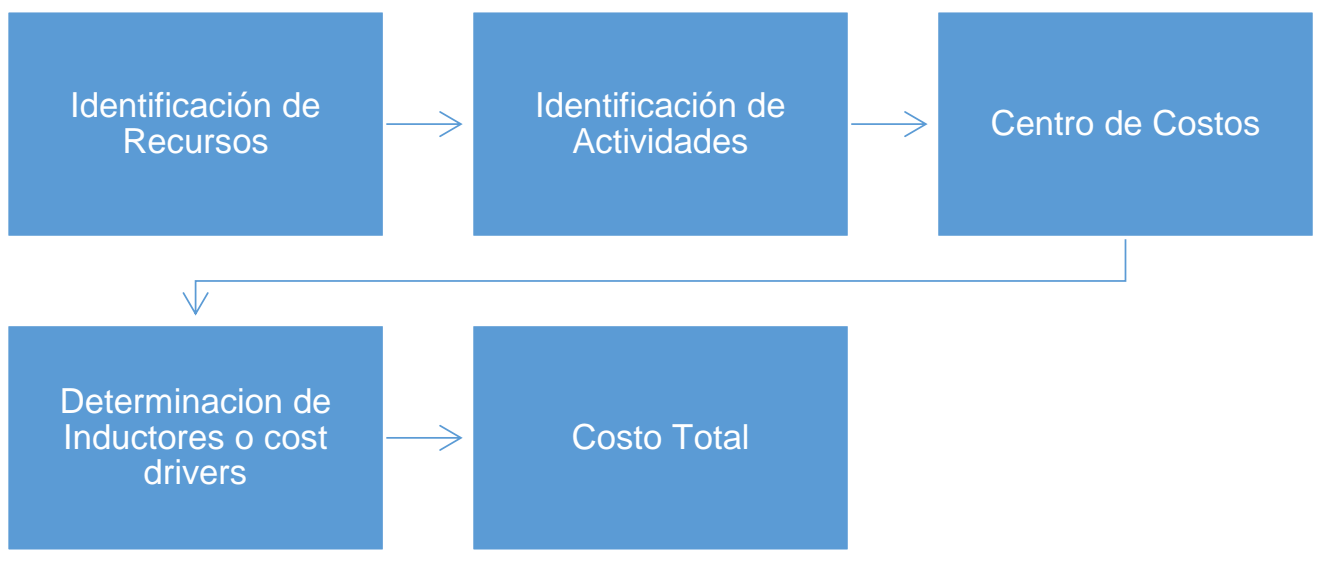

Fuente: Baldera (2016)

- Identificación de Recursos.

Los recursos son todos los elementos que se utilizan o se consumen en el desarrollo de las actividades. El sistema de costos $\mathrm{ABC}$ tiene como objetivo presentar los costos de un bien o servicio real considerando los materiales, la mano de obra, los materiales indirectos y todos los costos de las actividades necesarias para la producción. Así mismo, se deben considerar las actividades operativas realizadas por las áreas de apoyo (Cuervo, Osorio, y Duque, 2016). No obstante, no se consideran como un costo los gastos financieros, otros gastos administrativos y de otras áreas que no participan con el proceso productivo y los gastos donde no existe una erogación de dinero, tal como la depreciación de la propiedad, planta, equipo, maquinaria, entre otros.

- Identificación de Actividades.

Las actividades se definen como la secuencia de tareas para lograr un objetivo. En este escenario, Rodríguez (2008) clasifica las actividades directas e indirectas o también conocidas como de apoyo. Las actividades directas son aquellas que benefician directamente a la producción; mientras que las actividades de apoyo se identifican como el conjunto de tareas relacionadas, pero que no son parte del proceso productivo, por ejemplo: actividades realizadas por la parte administrativa.

Por otra parte, Möller (2011) afirma que las actividades se clasifican de acuerdo a lo que se quiere analizar: a) en función de la actuación con respecto al producto, b) según el ámbito de acción, c) en función de su capacidad para añadir valor al producto o servicio, d) según la frecuencia de ejecución, e) según la función o área a la que estén adscritas, f) por su incidencia en la consecución de los factores clave de éxito. 
- Creación y asignación de drivers o inductores de Recursos.

Para garantizar la confiabilidad del modelo $\mathrm{ABC}$, es necesario trabajar con inductores, (también conocidos como conductores o drivers) para que se cumpla con el propósito de brindar información fiable, confiable y relevante.

En este aspecto, un inductor de costo es un mecanismo de medida que presenta el costo de una actividad de forma precisa, permitiendo la asignación moderada de los costos a los productos o servicios. Una de las principales características de los inductores de costos es que vinculan las actividades con los productos, buscando una relación entre el consumo de factores y la producción alcanzada. Luego, identifican cada actividad de acuerdo a su función de mayor a menor y permiten distinguir posibles opciones de mejora (Mölller, 2011). Los inductores son considerados factores de asignación o distribución que se eligen porque identifican de la mejor manera como el costo es consumido.

- Centro de Costos

El centro de costos es el lugar en donde se incorporan los elementos de costos con las actividades, esta información se puede obtener de dos formas; la primera considera la naturaleza del elemento del costo y agrupando con las actividades, la segunda es mediante el análisis de los informes y documentación de lo que ha se ha consumido en los diferentes departamentos para ciertas actividades (Bustamante, 2015). Es importante mencionar que, una vez identificado los elementos del costo, las actividades, los recursos que necesitan las actividades y subprocesos; se deben multiplicar los inductores de asignación de los costos por el valor del recurso obteniendo como resultado del costo de la actividad y finalmente del costo total.

\section{ABM (Activity Based Management)}

El ABM es una herramienta para la toma de decisiones, que permite a la administración de una empresa mejorar a través de la disminución de los costos e incremento de la eficiencia, desarrollando ventajas competitivas y alcanzando metas de rentabilidad. El ABM, no solamente se enfoca en el logro de la excelencia empresarial, sino también, en el sostenimiento de la misma, exigiendo una mejora continua de todas las actividades de la empresa, tanto de las áreas productivas como la administrativa. Es así que, el ABC evalúa la viabilidad, mientras que el ABM garantiza la viabilidad (Cuervo, Osorio, y Duque, 2016). Los costos $\mathrm{ABC}$ se relacionan con el ABM al tratar la asignación de los costos por actividades y decidir sobre aspectos tales como: la reducción de costos, la dirección o planificación estratégica, la merma de desperdicios, el desarrollo de acciones competitivas, el manejo de forma adecuada de la estrategia de precios, y la generación de información que permita elegir si desarrollar o comprar un bien.

Por tanto, el ABM se enfoca en la gestión y excelencia de las empresas mientras que, el ABC alcanza sus objetivos a través de dos subcomponentes adjuntos dentro del ABM: operativo y 
estratégico. El ABM operativo se refiere al mejoramiento continuo de las actividades de una organización, engloba las gestiones que incrementan la eficiencia, reducen costos y mejoran la utilización de los recursos; realiza una reingeniería de procesos, busca la calidad total en todas las actividades y aumenta la eficiencia de los recursos de la empresa; lo que lleva a su vez, a obtener costos reducidos e ingresos mayores y menos costosos. Mientras que, el ABM estratégico intenta realizar un cambio en las actividades para incrementar la rentabilidad, asumiendo que la eficiencia de la actividad sigue siendo constante (Golpe, 2017).

El ABM como se mencionó en párrafos anteriores, es una herramienta para la toma decisiones por lo que es necesario comprender y entender la definición de las decisiones y su clasificación. En tal sentido, las decisiones son aquellas situaciones en el que un sujeto o individuo tiene dos alternativas, opciones o formas para resolver la misma, por otro lado, las alternativas no siempre dan resultados positivos o negativos, se podrá decir que es un riesgo al que está sujeto cada individuo al momento de tomar una decisión. Por ello, la toma de decisiones debe ser razonada y planificada. Por lo general las decisiones se toman con el objetivo de buscar un resultado positivo para lograr el éxito y no llegar al fracaso (Fundación para el desarrollo Socioeconómico y Restauración Ambiental, 2011).

Solano (2003) indica que las alternativas deben ser analizadas y evaluadas para determinar las ventajas y desventajas, después se debe identificar las soluciones a esas alternativas y con ello garantizar que los resultados sean los esperados y que reflejen validez y efectividad. Por lo antes anotado, se debe considerar la importancia de las decisiones, es decir ubicarles de forma abstracta en un orden de prioridad y peso, lo que permite a un individuo que le dedique más tiempo y recursos para aquella decisión que dependerá o involucra más factores. Por ejemplo, la decisión de comprar herramientas (decisión ligera e irrelevante), mientras si desea comprar una empresa (es una decisión que involucra más factores lo cual será necesario un análisis de la situación para poder decidir). En función a ello, las decisiones se clasifican en estratégicas, tácticas y operacionales.

En virtud de ello, las decisiones estratégicas son tomadas por los altos directivos, quienes ponen en consideración los fines, objetivos, misión y visión de la organización que dirigen. Además, toman en consideración otros factores, como tiempo, calidad y costos. En consecuencia, la información a tratar debe ser eficiente, oportuna y de calidad ya que si se toma una mala decisión estaría en riesgo toda la organización (Canós, Pons, Valero, y Maheut, 2012). De igual manera, las decisiones tácticas o también llamadas de pilotaje son aquellas tomadas por la dirección media de la empresa u organización, la cual se encarga de ejecutar las decisiones estratégicas, utilizando los recursos de forma racionalizada para evitar el desperdicio de recursos. Un error en este nivel no afectaría a toda la organización (Universidad de Jaén, 2005). Mientras que, las decisiones operativas se tratan en los niveles jerárquicos más bajos tales como los supervisores o jefes departamentales. En este nivel se encargan de las decisiones relacionadas con las funciones rutinarias de la empresa u 
organización. De modo que, si se comete un error, éste se podría considerar como leve ya que no implicaría cambios muy fuertes (ibídem).

Figura 2. Tipos de decisiones

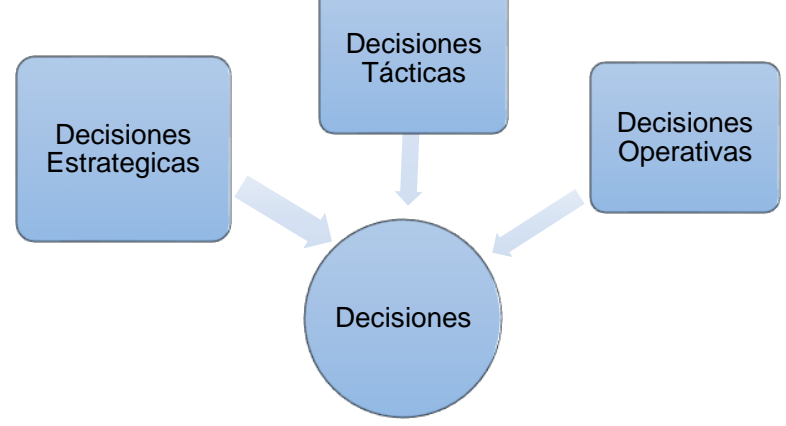

Fuente: Canós, Pons, Valero, y Maheut (2012)

\section{Metodología.}

Hernández y Baptista (2014) afirman que el enfoque mixto de la investigación implica un proceso de recolección, análisis y vinculación de datos cualitativos y cuantitativos en un mismo estudio o una serie de investigaciones para responder a un planteamiento del problema. Por lo antes expuesto, la presente investigación se enmarco en un enfoque mixto donde se empleó herramientas cualitativas para elaborar las bases teóricas y cuantitativas para valorar los datos que se obtuvieron en el diagnóstico de la empresa, con un alcance descriptivo-explicativo.

Los métodos empleados en la investigación fueron: el histórico-lógico que permitió analizar las variables de estudio bajo un orden cronológico y con una secuencia lógica, además se empleó el método deductivo-inductivo, este par dialectico posibilitó la identificación de procesos y actividades en la empresa objeto de estudio; finalmente se aplicó el método para el tratamiento de la literatura consultada

En lo referente a las técnicas e instrumentos se utilizaron encuestas aplicada de manera estratificada mediante cuestionarios a los socios y personal de los departamentos de contabilidad y técnico. Se utilizó la entrevista estructurada para obtener información a profundidad sobre las decisiones estratégicas de la empresa. En virtud de lo mencionado, se consideró como universo de estudio a 14 personas (tabla 1) que se encuentran relacionada directa e indirectamente con la determinación de los costos y la toma de decisiones 
Tabla 1. Población PlanERP.

\begin{tabular}{cccc}
\hline & Área & Cargo & N. Personas \\
\hline & Dirección & Accionistas & 3 \\
PlanERP CIA. LTDA. & Administración & Gerente & 1 \\
& Contabilidad & Área contable & 2 \\
& Técnica & Desarrolladores & 8 \\
& & Total & $\mathbf{1 4}$ \\
\hline
\end{tabular}

Fuente: PlanERP Cía. Ltda.

\section{Resultados.}

Los resultados de la aplicación de los instrumentos de investigación para el análisis de los costos en PlanERP Cía. Ltda., se muestran a continuación:

$\checkmark$ La empresa PlanERP actualmente cuenta con un sistema de costeo tradicional, el mismo que no presenta información confiable, oportuna y acertada de los costos de cada proyecto de implementación del software, provocando que la administración tome decisiones poco acertadas.

$\checkmark$ Se evidencio que no existe un adecuado reconocimiento de los elementos del costo, lo que ocasiona que los costos reflejados en los estados financieros sean incompletos y parte de los costos estén considerados como un gasto, provocando un costo de venta irreal y presentando un margen de rentabilidad confuso y poco veraz.

$\checkmark$ Existe un desconocimiento por parte del personal sobre los procesos de implementación del software (Sistema de Información Integral Multifinalitario SIIM), lo que conlleva a la duplicidad de funciones y sobre estimación de los tiempos requeridos para el cumplimiento de actividades.

$\checkmark$ No se realiza un plan de control para el personal, aun cuando cuenta con herramientas de control de actividades, esto da paso a que en ocasiones existan retrasos en los tiempos de implementación del software y una incorrecta asignación de horas hombre a los diferentes proyectos.

$\checkmark$ Se evidenció que la información contable presentada a la administración es poco confiable debido al deficiente sistema de costeo que aplica la empresa

A partir de los resultados obtenidos en la fase de diagnóstico se considera pertinente presentar a los directivos de la empresa PlanERP un modelo de gestión de costos ABC/ABM, que contribuya a la acertada toma de decisiones. Bajo este contexto, se diseña la propuesta partiendo de la identificación de: los servicios prestados por la empresa, las actividades que agregan valor, las personas involucradas, los elementos de costo consumidos y los inductores 
de asignación de los mismos. El modelo propuesto contempla cinco componentes: elementos del costo, identificación de actividades, centro de costos, determinación de inductores, y por último la determinación del costo total con el fin de mejorar la gestión de los costos de la empresa.

\section{ABM - Estudio y clasificación de procesos}

Los procesos de una empresa deben ser enmarcados con la visión, misión y objetivos de la empresa, para lograr la eficiencia; a esto se le conoce como gestión por procesos, este planteamiento viene a reemplazar a la gestión tradicional basada en las funciones o puestos de trabajo, para ello se plantea que los procesos deben ser sistemáticos y estratégicos.

Figura 3. Modelo de costeo ABC/ABM para la empresa PlanERP

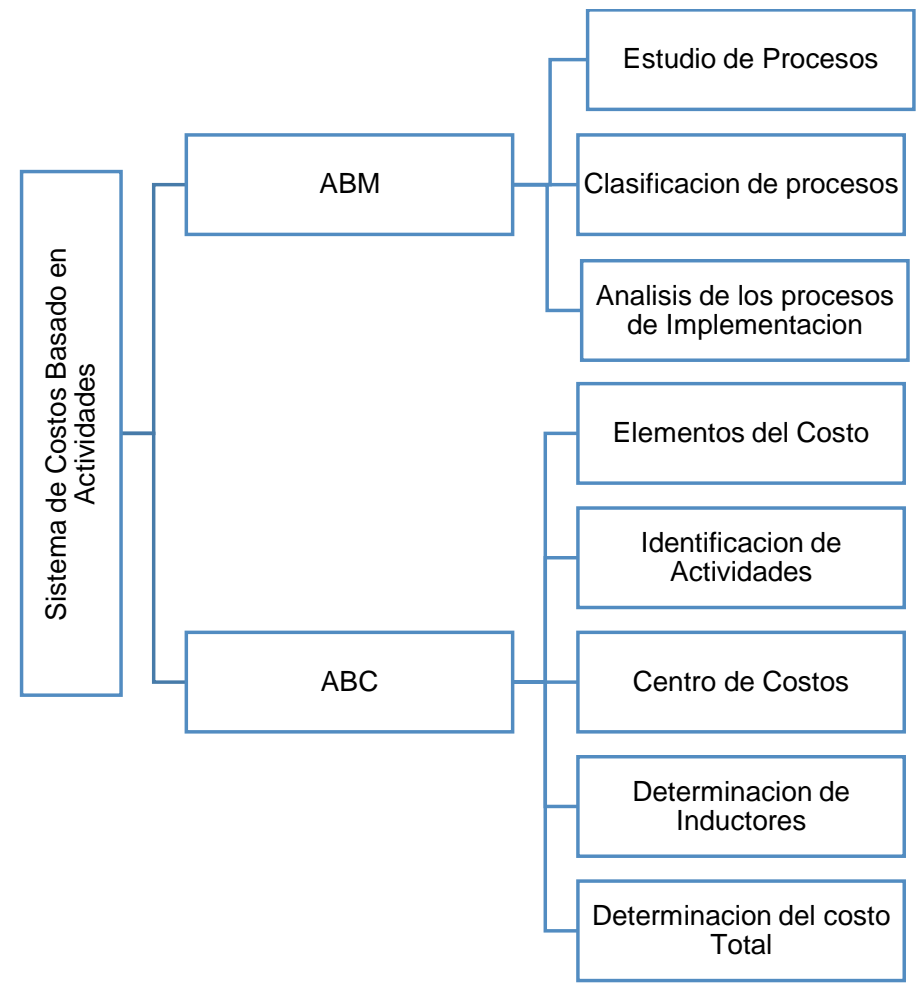

Fuente: Elaboración propia.

\section{$\checkmark \quad$ Procesos estratégicos o corporativos}

Los procesos estratégicos están íntimamente relacionados con la planificación, organización, dirección, evaluación y control, es decir, proporciona las directrices para los demás procesos tanto; el encargado de este proceso es la administración de la empresa PlanERP. 


\section{$\checkmark \quad$ Procesos operativos o de negocios.}

Los procesos operativos son aquellos realizados por el departamento técnico, es decir quienes se encargan de la implementación del software en los diferentes clientes de la empresa PlanERP.

\section{$\checkmark \quad$ Procesos de apoyo o soporte.}

Los procesos de apoyo son aquellos que no están relacionados directamente con la implementación del software, pero son necesarios para que se dé el proceso de implementación, como por ejemplo es necesario la intervención de la gerencia, el marketing y publicidad del software y más áreas necesarias.

Los procesos se clasificarán de acuerdo a su propósito, es por ello, que lo clasificaremos en estratégicos, operativos y de ayuda o apoyo.

Figura 4. Clasificación de procesos
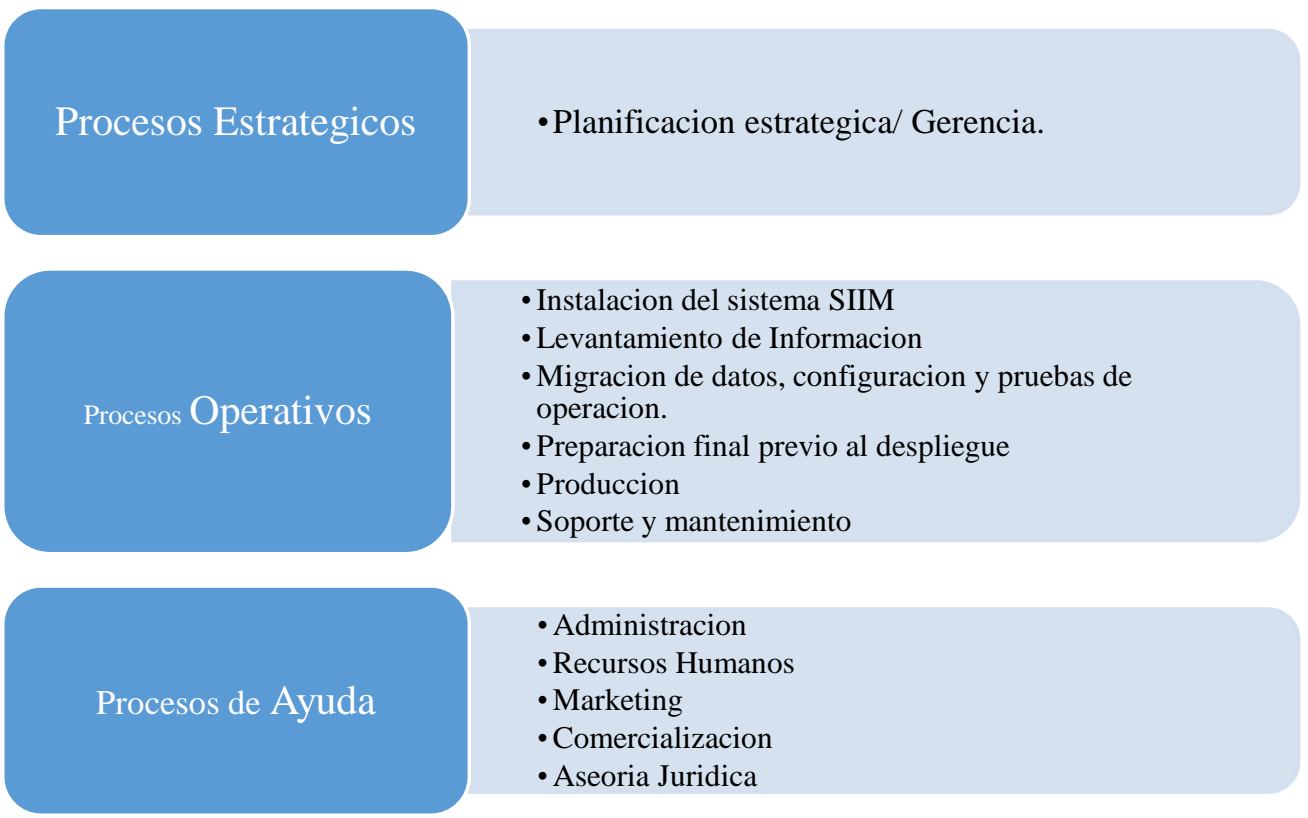

Fuente: Elaboración propia.

\section{Análisis de procesos de la empresa}

\section{Procesos estratégicos}

La gerencia considerando la información obtenida de las diferentes áreas determina cómo asignar los recursos económicos y humanos y cómo se vincula con las operaciones de la empresa. Además, hace frente a problemas legales de cualquier naturaleza; ya sea, por contratos con clientes, seguros (pólizas) y/o casos tributarios. 
Así también, dentro de los procesos estratégicos se encuentra la gestión de recursos humanos donde se elaboran pruebas de admisión: psicológicas y técnicas para el reclutamiento, pero además cuestionarios de evaluación y medición del desempeño del personal mediante herramientas de control de tiempos de trabajo.

\section{Procesos operativos}

Levantamiento de información de clientes. Elaboración de planes de trabajo de acuerdo a los plazos estipulados, solicitar documentación tales como: leyes, ordenanzas, resoluciones, políticas y fórmulas de cálculo que permita conocer el manejo y flujos de procesos de la entidad; ya que esto posteriormente se implementara y/o parametrizara en el sistema.

Migración de datos. Se solicita la base de datos del sistema anterior; es decir, la información con que cuenta la entidad para iniciar con el análisis, extracción, transformación y carga de datos al sistema SIIM.

Instalación del sistema SIIM, configuración y pruebas de operación: Instalación del sistema SIIM en el servidor de la entidad, parametrización del sistema, aplicación de lo estudiado en los documentos, leyes, resoluciones, etc.

Preparación final previo al despliegue: Levantamiento de actas sobre las pruebas funcionales y no funcionales por parte de los usuarios expertos encargados de cada módulo del sistema, levantamiento de actas del proceso de capacitación a los usuarios finales del sistema y capacitación técnica a los departamentos de sistemas de los clientes o entidades.

Producción: El sistema SIIM, inicia en operación.

Soporte y mantenimiento: Firma de garantía técnica que incluye un año de soporte y mantenimiento, una vez se haya firmado la entrega del producto contratado. El soporte aplicara corrección de errores sobre la programación del sistema.

\section{Procesos de ayuda}

La empresa cuenta con un profesional especializado en publicidad y marketing, ya que en la actualidad existen avances tecnológicos como las redes sociales, páginas web y sitios virtuales que son herramientas útiles para publicitar el sistema SIIM.

\section{Estructura Organizativa.}

Una vez detallados los procesos de PlanERP Cía. Ltda., es oportuno conocer quién es responsable de cada proceso y determinar un nuevo organigrama para la empresa, el mismo que se muestra en la Figura 5, donde se ha incluido un Asesor Legal y un departamento de comercialización que es lo que carece la empresa. 
Figura 5. Organigrama

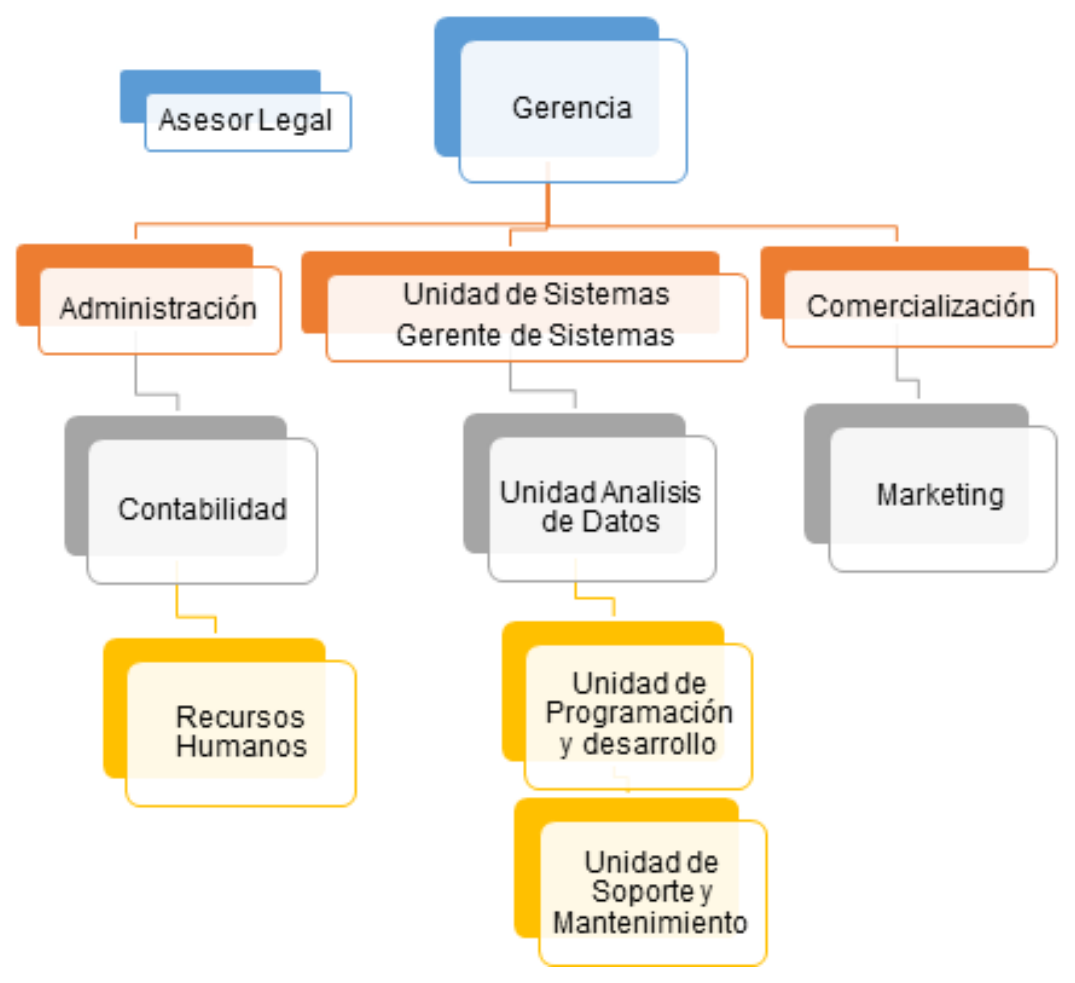

\section{Costeo ABC}

\section{$\checkmark \quad$ Elementos del costo.}

Los elementos del costo son aquellos insumos, materiales y recursos humanos que se consumen en el periodo para la prestación del servicio. Una vez que se conozcan estos podrán ser asignados a los diferentes procesos y tener oportunamente información de cómo y para qué estos fueron consumidos. Por lo tanto, los elementos del costo identificados en la empresa PlanERP se detallan a continuación:

Tabla 2. Elementos del costo Implementación del Sistema SIIM.

\begin{tabular}{ll}
\hline Codificación & \multicolumn{1}{c}{ Elementos del costo } \\
\hline C1 & Licencia del Software Sistema Integral de Información Multifinalitario \\
C2 & Sueldos y salarios del personal técnico \\
C3 & Seguridad Social a cargo de la empresa \\
C4 & Primas de Seguro \\
C5 & Transporte \\
C6 & Viáticos \\
C7 & Depreciación Equipos de Computo \\
C8 & Arriendos \\
C9 & Marketing y Publicidad \\
C10 & Electricidad \\
C11 & Agua
\end{tabular}




$\begin{array}{ll}\text { C12 } & \text { Internet } \\ \text { C13 } & \text { Telefonía } \\ \text { C14 } & \text { Depreciación vehículos } \\ \text { C15 } & \text { Insumos de Oficina } \\ \text { C16 } & \text { Sueldos y salarios del personal administrativo } \\ \text { C17 } & \text { Seguridad Social a cargo de la empresa administrativo }\end{array}$

\section{$\checkmark \quad$ Identificación de Actividades.}

Las actividades que han sido identificadas en el proceso de implementación del sistema SIIM se detallan en la Tabla 3.

Tabla 3. Elementos del costo Implementación del Sistema SIIM.

\begin{tabular}{ll}
\hline ID & Identificación de Actividades \\
\hline A1 & Preparación del proyecto e instalación del SIIM \\
A1.1 & Actividades de gestión del proyecto \\
A1.2 & Preparación del kick-off del proyecto \\
A1.3 & Reunión de kick-off \\
A1.4 & Entrega e instalación del software \\
A2 & Levantamiento de información \\
A2.1 & Actividades de gestión del proyecto \\
A2.2 & Sesiones de requerimientos de la entidad \\
A2.3 & Revisar el documento de características de la entidad \\
A3 & Migración de Datos, configuración y pruebas de operación \\
A3.1 & Gestión general del proyecto \\
A3.2 & Configuración del sistema en producción \\
A3.3 & Gestión del cambio organizacional \\
A3.4 & Pruebas de validación/aceptación del sistema \\
A3.5 & Pruebas del sistema \\
A3.6 & Planificar salida a producción y soporte \\
A4 & Preparación final previo despliegue en producción \\
A4.1 & Gestión general del proyecto \\
A4.2 & Entrenamiento \\
A4.3 & Preparación del sistema \\
A4.4 & Transición \\
A5 & Despliegue en producción \\
A5.1 & Soporte en producción \\
A5.2 & Mejora continua \\
A5.3 & Cierre del proyecto \\
A6 & Soporte técnico \\
A6.1 & Garantía técnica \\
\hline & \\
\hline
\end{tabular}




\section{Centro de Costos}

El centro de costos es el lugar en donde se incorporan los 17 elementos de costos identificados con las 6 actividades principales y subprocesos.

Tabla 4. Centro de Costos PlanERP Cía. Ltda.

\section{Elementos de Costo}

\section{Actividades para la Implementación \\ Sistema Integral de Información} Financiera SIIM

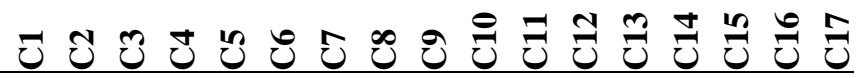

- Preparación del Proyecto e

Instalación del SIIM

¿ Actividades de Gestión del Proyecto

$\stackrel{\sim}{\gtrless}$ Preparación del kick-off del Proyecto

$\stackrel{n}{\gtrless}$ Reunión de Kick-off

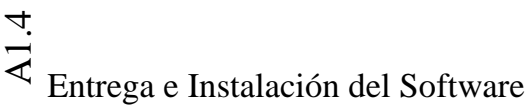

\ Levantamiento de información

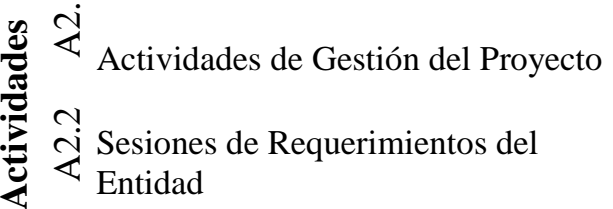

กี Revisar el Documento de

Características del Entidad

ఇ Migración de Datos, Configuración y Pruebas de Operación

ஓं Gestión General del Proyecto

ণั Configuración del Sistema en

$\varangle$ Producción

$\stackrel{m}{\gtrless}$ Gestión del Cambio Organizacional

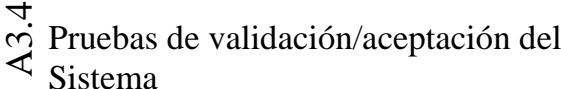

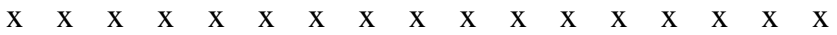

$\begin{array}{llllllllllllllll}\mathrm{X} & \mathrm{X} & \mathrm{X} & \mathrm{X} & \mathrm{X} & \mathrm{X} & \mathrm{X} & \mathrm{X} & \mathrm{X} & \mathrm{X} & \mathrm{X} & \mathrm{X} & \mathrm{X} & \mathrm{X} & \mathrm{X} & \mathrm{X}\end{array}$

$\begin{array}{lllllllllllllllll}\mathrm{X} & \mathrm{X} & \mathrm{X} & \mathrm{X} & \mathrm{X} & \mathrm{X} & \mathrm{X} & \mathrm{X} & \mathrm{X} & \mathrm{X} & \mathrm{X} & \mathrm{X} & \mathrm{X} & \mathrm{X} & \mathrm{X} & \mathrm{X}\end{array}$

$\begin{array}{llllllllllllllll}\mathrm{X} & \mathrm{X} & \mathrm{X} & \mathrm{X} & \mathrm{X} & \mathrm{X} & \mathrm{X} & \mathrm{X} & \mathrm{X} & \mathrm{X} & \mathrm{X} & \mathrm{X} & \mathrm{X} & \mathrm{X} & \mathrm{X} & \mathrm{X}\end{array}$

$\begin{array}{lllllllllllllllll}X & X & X & X & X & X & X & X & X & X & X & X & X & X & X & X & X\end{array}$

$\begin{array}{llllllllllllllll}\mathrm{X} & \mathrm{X} & \mathrm{X} & \mathrm{X} & \mathrm{X} & \mathrm{X} & \mathrm{X} & \mathrm{X} & \mathrm{X} & \mathrm{X} & \mathrm{X} & \mathrm{X} & \mathrm{X} & \mathrm{X} & \mathrm{X} & \mathrm{X}\end{array}$

$\begin{array}{llllllllllllllll}\mathrm{X} & \mathrm{X} & \mathrm{X} & \mathrm{X} & \mathrm{X} & \mathrm{X} & \mathrm{X} & \mathrm{X} & \mathrm{X} & \mathrm{X} & \mathrm{X} & \mathrm{X} & \mathrm{X} & \mathrm{X} & \mathrm{X} & \mathrm{X}\end{array}$

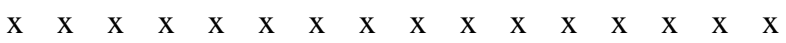

$\begin{array}{llllllllllllllll}X & X & X & X & X & X & X & X & X & X & X & X & X & X & X & X\end{array}$

$\begin{array}{llllllllllllllll}\mathrm{X} & \mathrm{X} & \mathrm{X} & \mathrm{X} & \mathrm{X} & \mathrm{X} & \mathrm{X} & \mathrm{X} & \mathrm{X} & \mathrm{X} & \mathrm{X} & \mathrm{X} & \mathrm{X} & \mathrm{X} & \mathrm{X} & \mathrm{X}\end{array}$

$\begin{array}{llllllllllllllllll} & X & X & X & X & X & X & X & X & X & X & X & X & X & X & X & X\end{array}$

$\begin{array}{llllllllllllllll}\mathrm{X} & \mathrm{X} & \mathrm{X} & \mathrm{X} & \mathrm{X} & \mathrm{X} & \mathrm{X} & \mathrm{X} & \mathrm{X} & \mathrm{X} & \mathrm{X} & \mathrm{X} & \mathrm{X} & \mathrm{X} & \mathrm{X} & \mathrm{X}\end{array}$

$\begin{array}{llllllllllllllll}X & X & X & X & X & X & X & X & X & X & X & X & X & X & X & X\end{array}$ 
年

Pruebas del Sistema

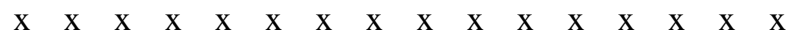

$\stackrel{\sigma}{\gtrless}$ Planificar Salida a Producción y
Soporte

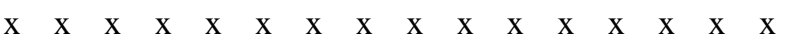

¿ Preparación Final previo Despliegue

en Producción

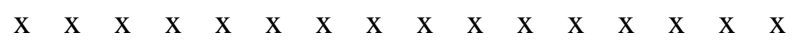

$\stackrel{+}{\longleftarrow}$

Gestión General del Proyecto

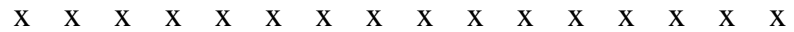

$\stackrel{\Upsilon}{\longleftarrow}$ Entrenamiento

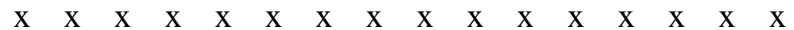

$\stackrel{n}{\longleftarrow}$ Preparación del Sistema

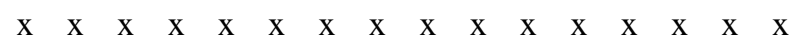

$\stackrel{\forall}{ষ}$ Transición

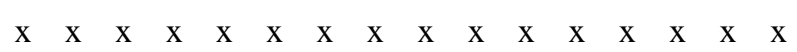

$\stackrel{\text { in }}{\ll}$ Despliegue en Producción

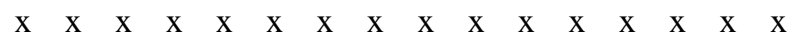

in

Soporte en Producción

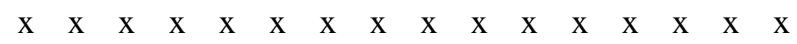

ก!

Mejora Continua

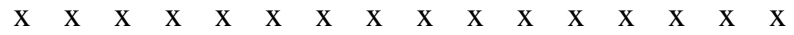

発 Cierre del proyecto

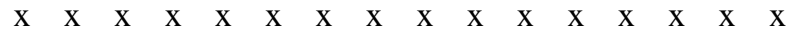

光 Soporte Técnico

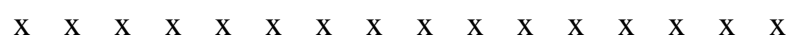

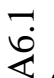

Garantía Técnica (1 año gratuito)

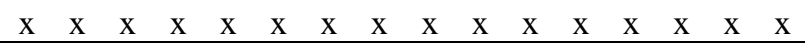

\section{$\checkmark \quad$ Determinación de los inductores de coste}

Al tener identificadas las actividades y subprocesos, es momento de seleccionar los inductores y el comportamiento de los mismos. 
Tabla 5. Asignación de Inductores

\begin{tabular}{lll}
\hline Codificación & \multicolumn{1}{c}{ Elementos del Costo } & \multicolumn{1}{c}{ Inductores } \\
\hline C1 & Licencia del Software Sistema Integral de Información Multifinalitario & Unidad \\
C2 & Sueldos y salarios del personal técnico & Horas Hombre \\
C3 & Seguridad Social a cargo de la empresa & Horas Hombre \\
C4 & Primas de Seguro & Unidad \\
C5 & Transporte & Horas Hombre \\
C6 & Viáticos & número de días \\
C7 & Depreciación Equipos de Computo & Horas uso \\
C8 & Arriendos & Horas Hombre \\
C9 & Marketing y Publicidad & Horas Hombre \\
C10 & Electricidad & Horas Hombre \\
C11 & Agua & Horas Hombre \\
C12 & Internet & Horas Hombre \\
C13 & Telefonía & Horas Hombre \\
C14 & Depreciación Vehículos & Horas Hombre \\
C15 & Insumos de Oficina & Horas Hombre \\
C16 & Sueldos y salarios del personal administrativo & Horas Hombre \\
C17 & Seguridad Social a cargo de la empresa administrativo & Horas Hombre \\
\hline
\end{tabular}

\section{$\checkmark \quad$ Determinación del coste}

Una vez que todos los procesos han sido identificados, las actividades han sido detalladas y los recursos para cada uno de estos han sido asignados, se ha realizado un análisis de costos considerando los inductores mencionados en la sección anterior.

El sistema de costos $\mathrm{ABC}$ puede considerar los costos presupuestados, históricos o estándar, dependiendo de la información con la que cuente la empresa. Para la presente propuesta se considera los costos históricos, que tiene disponible la empresa para el análisis.

En la tabla 6 se observa la asignación de los elementos del costo $\mathrm{C} 1$ a C7 a las 6 actividades principales de la implementación del software.

Tabla 6. Determinación de los costes C1-C7

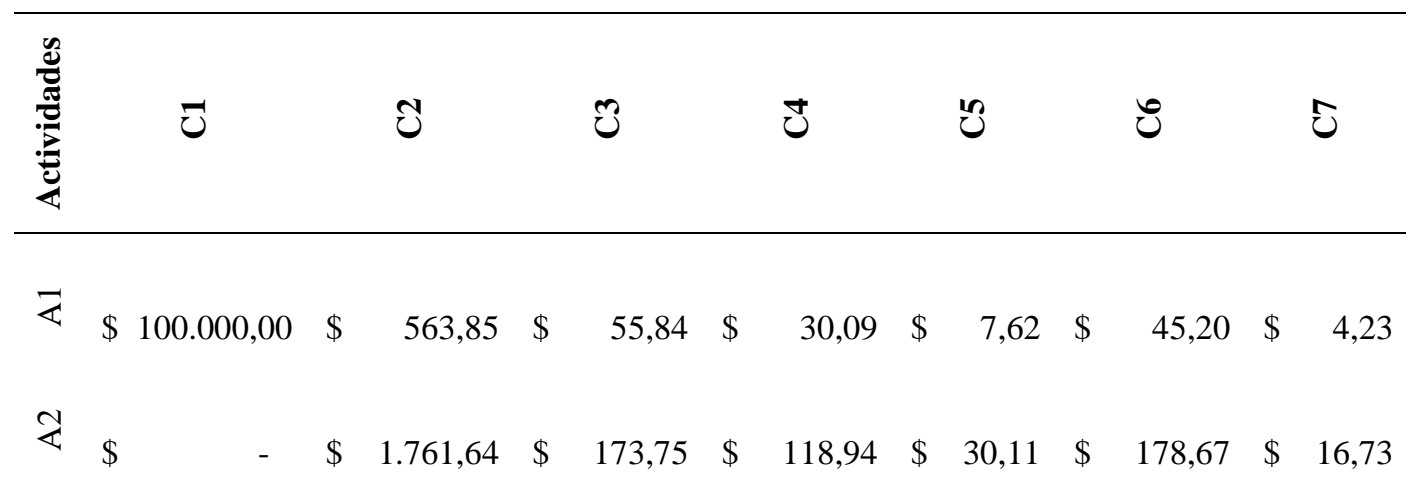




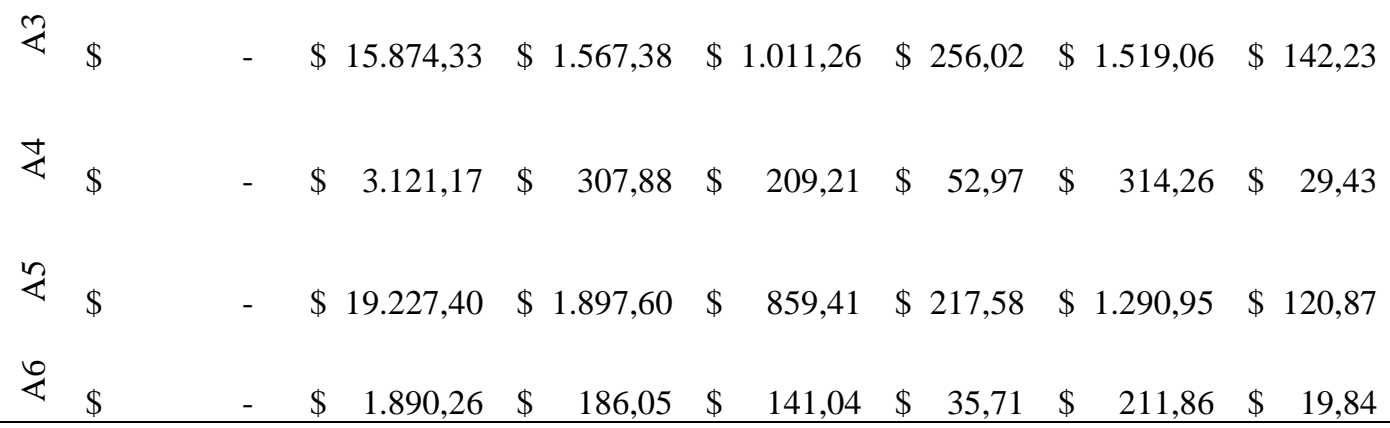

En la tabla 7 se observa la asignación de los elementos del costo C8 a C15 a las 6 actividades principales de la implementación del software.

Tabla 7. Determinación de los costos C8 a C15

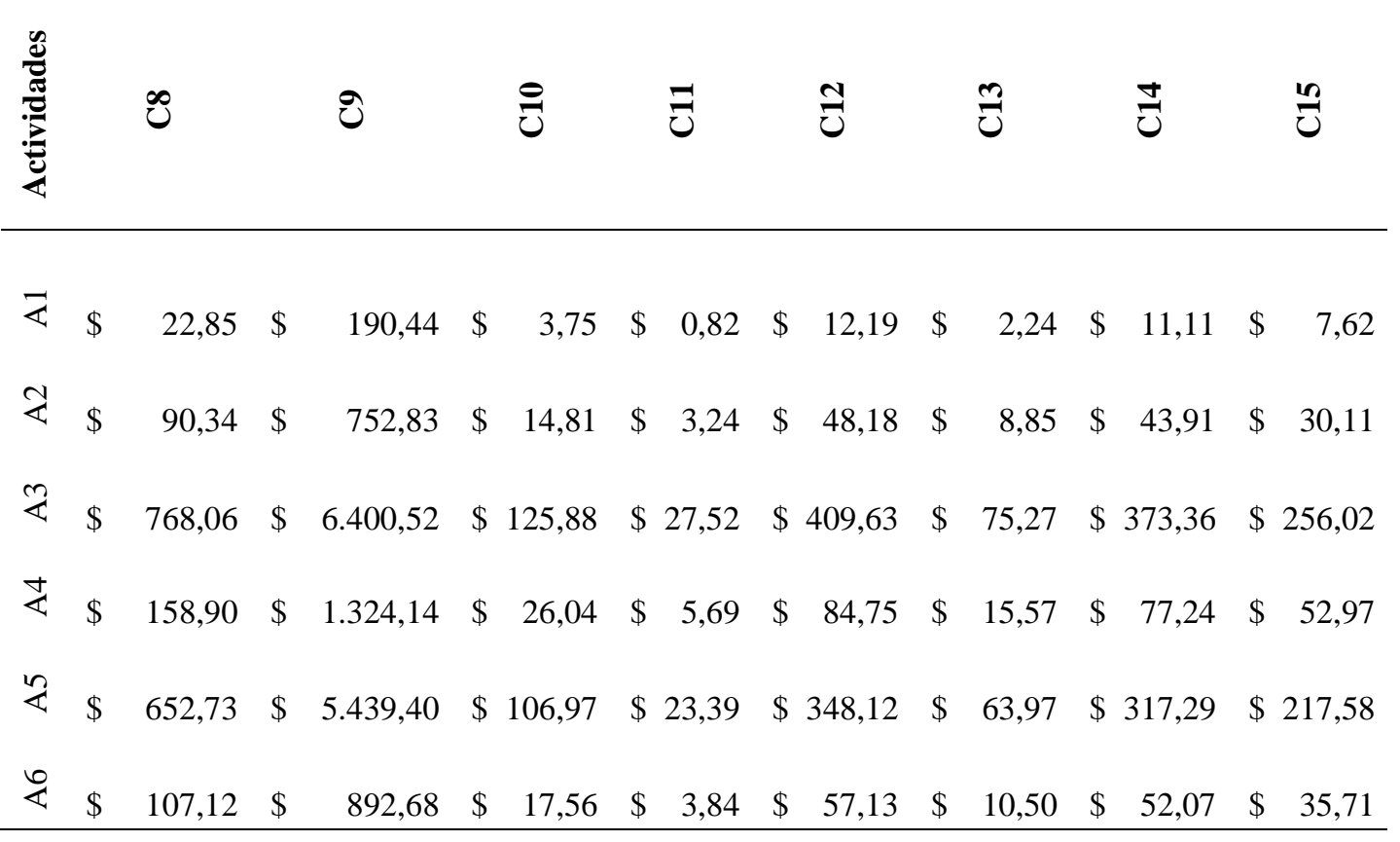

En la tabla 8 se observa la asignación de los elementos del costo C16 a C17 a las 6 actividades principales de la implementación del software, hasta determinar el costo por actividad.

Tabla 8. Determinación del costo C16-C17

\begin{tabular}{|c|c|c|c|c|c|c|}
\hline Actividades & & $\frac{v}{u}$ & & $\bar{y}$ & $\begin{array}{l}\text { Total costo } \\
\text { Actividad }\end{array}$ & $\%$ \\
\hline $\begin{array}{l}\text { Preparación del Proyecto e instalación } \\
\text { del SIIM }\end{array}$ & $\$$ & 49,54 & $\$$ & 4,86 & \$ 101.012,23 & $57 \%$ \\
\hline ¿ Levantamiento de información & $\$$ & 195,85 & $\$$ & 19,21 & $3.487,17$ & $2 \%$ \\
\hline
\end{tabular}


2. Migración de datos, configuración y

pruebas de operación

$\$ 1.665,13$

$\$ 163,31$

\$ $30.634,97$

$17 \%$

\& Preparación final previo despliegue en

$\begin{array}{lllllll}\$ & 344,48 & \$ & 33,79 & \$ & 6.158,48 & 3 \%\end{array}$

\&

Despliegue en producción

$\$ 1.415,09$

$\$ 138,79$

\$ $32.337,14$

$18 \%$

\&

Soporte Técnico

$\begin{array}{llllll}\$ \quad 232,24 & \$ & 22,78 & \$ & 3.916,38\end{array}$

$2 \%$

Una vez calculado los costos de las actividades, se aplica la siguiente fórmula para conocer el costo total de la Implementación del software:

$$
\text { Costo Total }=A 1+A 2+A 3+A 4+A 5+A 6
$$

Aplicando la fórmula:

$$
\begin{gathered}
\text { Costo Total }=\$ 101.012,23+\$ 3.487,17+\$ 30.634,97+\$ 6.158,48 \\
+\$ 32.337,14+\$ 3.916,38=\$ 177.546,37
\end{gathered}
$$

El principal costo es en la Actividad A1 preparación del proyecto e instalación del SIIM ocupando un $57 \%$ del costo total requerido en todo el proceso, un $18 \%$ se utiliza para el despliegue en producción (A5) y el $17 \%$ para la migración de datos (A3). Y apenas un $2 \%$ tanto para el levantamiento de información como para el soporte técnico una vez que el SIIM ha sido instalado, como se muestra en la Figura 6.

Figura 6. Representación de los costos en la implementación del software

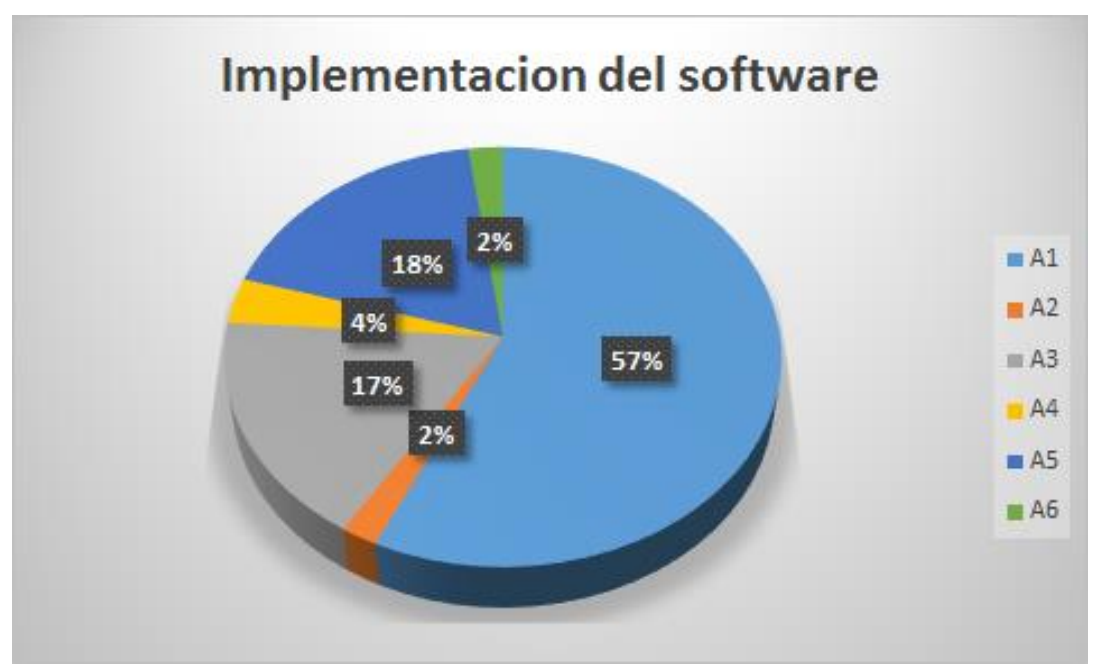

Este análisis permite identificar la actividad que utiliza más recursos y con ello un genera un mayor costo para de esta manera enfocarse en la misma y optimizar los recursos que consume, o reducir los recursos asignados en caso de ser necesario. Dentro de esta actividad 
se realiza un análisis más detallado como se muestra en la siguiente figura 7 lo cual indica que la actividad más costosa es la Entrega e instalación del SIIM que representa un 89\% del costo del total de esta actividad.

Figura 7. Costo por actividades

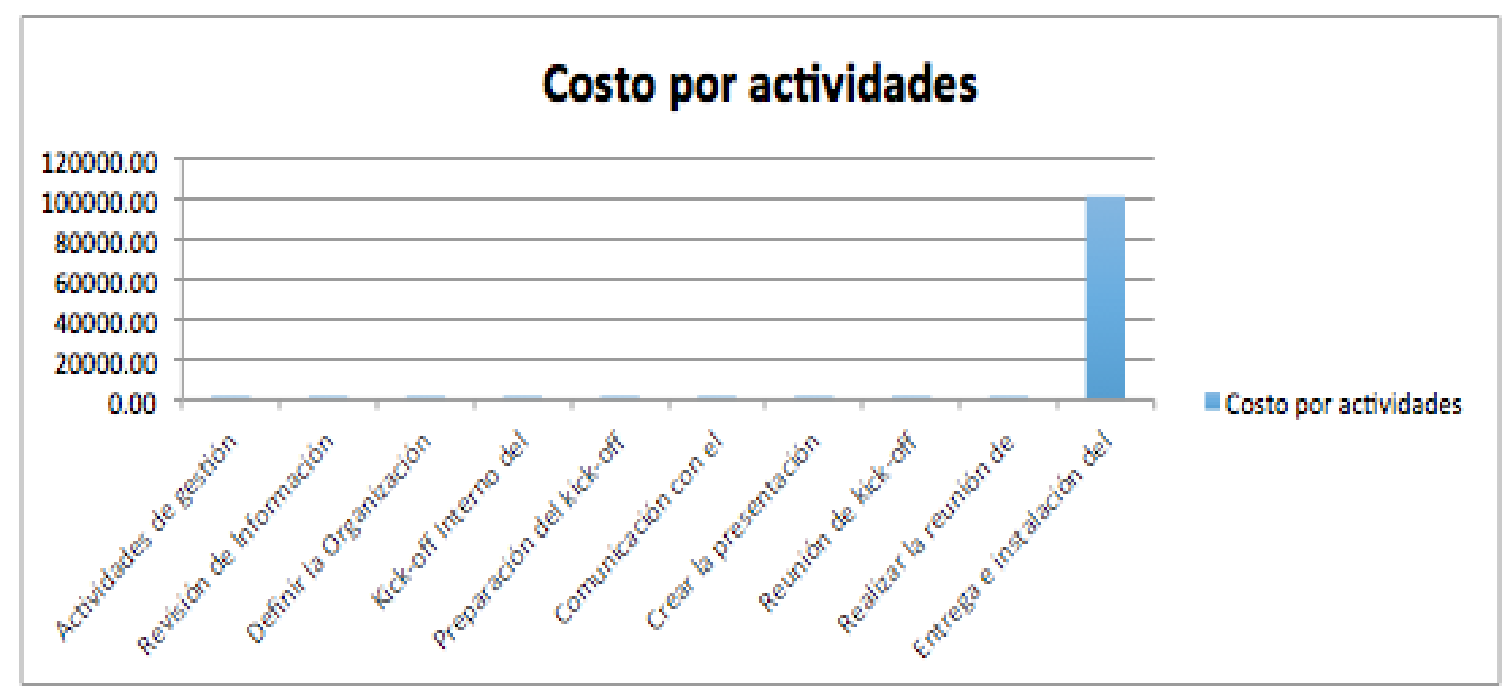

\section{Conclusiones}

$\checkmark \quad$ Existen diferentes métodos para calcular el costo de producción de un bien o servicio unos más cerca de la realidad, pero cada empresa debe analizar y estudiar cuál de los métodos son más apegados a la situación real del giro del negocio para obtener información más confiable y relevante, sin embargo, se puede decir que el sistema de costeo $\mathrm{ABC} / \mathrm{ABM}$ ha sido de gran utilidad para la empresa de software PlanERP porque permitió identificar los procesos más costosos que necesiten una optimización de los recursos, lo cual traerá más beneficios a la empresa.

$\checkmark \quad$ Las decisiones se deben tomar en todo momento ya sea en un problema o situación se debe elegir una alternativa como una posible solución, por ello, es necesario que se tome un tiempo para planificar y organizar cada una de las opciones que se presentan.

$\checkmark \quad$ Aplicar el sistema de costeo ABC/ABM ha permitido que la administración analice y defina los procesos que realiza y tenga un análisis de costos real, lo que conlleva a tomar mejores decisiones administrativas. El caos financiero puede reinar en una empresa que no tenga claro cuáles son sus costos, ya que se pueden omitir costos pequeños/medianos que interfieren durante todos los procesos, esto puede llevar a tomar decisiones poco acertadas en cuanto a los presupuestos de los próximos proyectos. 
$\checkmark \quad$ Las oportunidades para la transformación, reingeniería y mejoras continuas de los procesos pueden ser rápidamente identificadas por el sistema $\mathrm{ABC} / \mathrm{ABM}$, en el caso de la empresa PlanERP se pudo identificar que los tiempos para cada actividad no están bien definidos y por ende no se ejecutan con éxito y se generan retrasos en los proyectos lo que implica un mayor consumo de recursos e incremento de los costos.

\section{Referencias bibliográficas.}

Behar, D. (2008). Metodologia de la Investigación. Shalom.

Bustamante, A. (2015). Costeo Basado en Actividades-ABC Revision de Literatura. CEA, 109-119.

Canós, L., Pons, C., Valero, M., \& Maheut, J. (2012). Universidad Politécnica de Valencia. Obtenido de Toma de decisiones en la empresa: proceso y clasificación: https://riunet.upv.es/bitstream/handle/10251/16502/TomaDecisiones.pdf

Cuervo, Osorio, \& Duque. (2016). Costeo Basado en Actividades ABC Gestion Basada en Actividades ABM. Estados Unidos: EcoeEdiciones.

Fundación para el desarrollo Socioeconómico y Restauración Ambiental. (2011). ¿Como enseñar a tomar decisiones acertadas? San Salvador: Casa Barak. Obtenido de http://www.fundesyram.info/document/pdfpub/toma_decisiones.pdf

Golpe, A. M. (2017, November). La gestión estratégica de costos: ABC, ABM y la necesidad de otras clasificaciones de costos para la toma de decisiones. In Anais do Congresso Brasileiro de Custos-ABC.

Hernandez, R., Fernández, C., \& Baptista, P. (2014). Metodologia de la Investigación. Mexico: Miembro de la Cámara Nacional de la Industria Editorial Mexicana.

Martínez, R., \& Rodríguez, E. (2010). Manual de metodologia de la investigación Científica. Obtenido de http://www.sld.cu/galerias/pdf/sitios/cielam/manual_de_metodologia_deinvestigaci ones._1.pdf

Mayorga, C. (2015). Gerencia Estrategica de Costos. Ambato.

Mölller, G. (2011). El sistema de costos basado en las actividades (ABC) Implementacion en una bodega. Trabajo Fin de Master en Investigacion en Contabilidad y Gestion Financiera. Valladolid. Obtenido de https://uvadoc.uva.es/bitstream/10324/849/1/TFM118-110802.pdf 
Pérez, A. (06 de 2015). Diseño e implantación de un Modelo ABC/ABM para una Empresa Hotelera. Calculo de Costes e Indicadores de Gestión. Universidad de Málaga. Málaga, España.

Rodriguez, E. (febrero de 2008). "Activity Based Costing ABC" Costos Basados en Actividades, aplicacion a la gestion de una institucion financiera "Banca Universal". Tesis de Posgrado. Caracas, Venezuela. Obtenido de http://biblioteca2.ucab.edu.ve/anexos/biblioteca/marc/texto/AAR2324.pdf

Rodriguez, R., \& Macarro, M. (1996). El Sistema de Costos Basados en las Actividades (ABC). Un planeamiento Analítico. En R. Rodriguez González, \& M. J. Macarro Heredia, El Sistema de Costos Basados en las Actividades (ABC). Un planeamiento Analítico.

Solano, A. (2003). Dialneet-Toma de decisiones. Obtenido de https://dialnet.unirioja.es/descarga/articulo/4835719.pdf

Universidad de Jaén. (2005). Capitulo 5 Toma de Decisiones en la Empresa. Obtenido de http://www4.ujaen.es/ cruiz/diplot-5.pdf 


\section{PARA CITAR EL ARTÍCULO INDEXADO.}

Torres Flores, M. E. T. F., Narváez Zurita, C., Erazo Álvarez, J., \& Ormaza Andrade, J. (2019). Costos ABC/ABM como herramienta de gestión empresarial. Caso empresa PlanERP Cía. Ltda. Visionario Digital, 3(2.1.), 114-135.

https://doi.org/10.33262/visionariodigital.v3i2.1.549

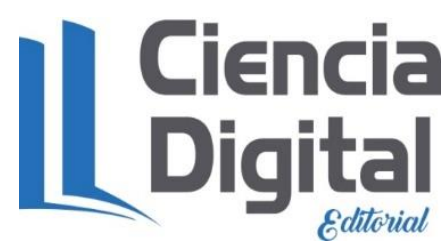

El artículo que se publica es de exclusiva responsabilidad de los autores y no necesariamente reflejan el pensamiento de la Revista Ciencia Digital.

El artículo queda en propiedad de la revista y, por tanto, su publicación parcial y/o total en otro medio tiene que ser autorizado por el director de la Revista Ciencia Digital.
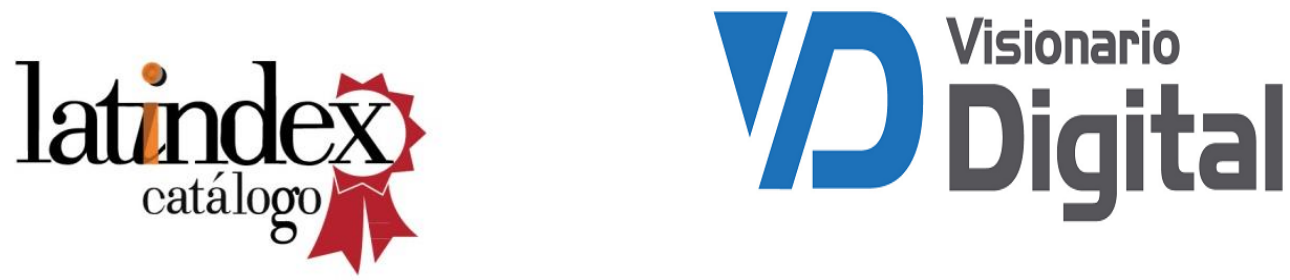